\title{
Development and evaluation of a colorimetric PCR system for the detection and typing of human papillomaviruses
}

\author{
DIEGO CHOUHY ${ }^{1,2}$, LISANDRO BENÍTEZ GIL ${ }^{3}$, ANA L. NOCITO ${ }^{4}$, DANIEL WOJDYLA ${ }^{5}$, \\ LEONARDO ORNELLA $^{1}$, JORGE CITTADINI ${ }^{3}$, DANIELA GARDIOL ${ }^{1,2}$ and ADRIANA A. GIRI ${ }^{1,2}$
}

\begin{abstract}
${ }^{1}$ Virology Area, School of Biochemistry and Pharmaceutical Sciences, Rosario National University, Suipacha 531, 2000 Rosario; ${ }^{2}$ Institute of Molecular and Cellular Biology of Rosario (IBR), National Council for Scientific and Technology Research (CONICET), Suipacha 531, 2000 Rosario; ${ }^{3}$ Division of Cervical Pathology, Centenario Provincial Hospital, Urquiza 3101, 2000 Rosario; ${ }^{4}$ Department of Pathology, School of Medicine, Rosario National University, Santa Fe 3100, 2000 Rosario; ${ }^{5}$ Biostatistics Area, School of Statistics, Rosario National University, Bv. Oroño 1261, Rosario, Argentina
\end{abstract}

Received April 14, 2006; Accepted June 15, 2006

\begin{abstract}
In developing countries, the introduction of human papillomaviruses (HPV) DNA testing as an adjunct to cytological screening programs has been delayed due to the lack of high performance and cost effective diagnostic nucleic acid methods. In this study we report the development and evaluation of the L1HPVPCR, a PCR-based method for the detection and typing of five of the most prevalent high-risk HPV types. The L1HPVPCR assay combines amplification with the MY09/11 HPV consensus primer system, liquid hybridization of the PCR products with no radioactive probes and enzyme immunoassay analysis. The technique is a userfriendly system that allows accurate HPV DNA detection and typing with inexpensive instrumentation that could be performed with not sophisticated reagents in almost any laboratory. Different cutoff points for generic and specific HPV detection were determined using reproducibility analysis and receiver operating characteristic curves to ensure good analytical sensitivity and clinical effectiveness. We used the L1HPVPCR assay to estimate the prevalence of HPV infection in 127 women at risk of cervical cancer from the city of Rosario (Argentina), where no epidemiological data has been previously reported. Further, we explored the clinical utility of the L1HPVPCR assay respect the Pap smear using a combined diagnosis of cytology, histology and colposcopy as gold standard. In conclusion, our results indicate that the assay described here provides a tool for accurate HPV DNA testing and could be applied in regions where no commercial tests are available.
\end{abstract}

Correspondence to: Dr Adriana A. Giri, Virology Area, School of Biochemistry and Pharmaceutical Sciences, Rosario National University, Suipacha 531, 2000 Rosario, Argentina

E-mail: agiri@fbioyf.unr.edu.ar

Key words: cervical cancer, developing countries, Argentina, HPV DNA testing, PCR-EIA assay

\section{Introduction}

Cervical cancer is considered the second most common cancer affecting women in the world (1). Globally, it is estimated that approximately 500,000 new cases of cervical cancer are reported each year, $80 \%$ of them in developing countries (2). Latin America and the Caribbean have some of the highest cervical cancer incidence and mortality rates in the world, being only surpassed by East Africa and Melanesia (3). Estimation in year 2000 showed Argentina as one of the countries with the lowest incidence $(14.2 / 100,000)$ and mortality $(7.2 / 100,000)$ rates in Latin America. However, these rates are elevated if compared with estimates for American developed countries such as USA (7.8/100,000 and 3.3/100,000, respectively) or Canada (8.2/100,000 and 2.8/100,000, respectively) (2).

Cervical cancer is a disease fully preventable and curable when screening of asymptomatic women is available, together with appropriate diagnosis, treatment and follow-up (3). Screening is traditionally based on periodic Papanicolaou smear (Pap smear) cytology. Precursors of invasive cervical carcinoma are staged according to increasing severity into low-grade (LSILs) and high-grade squamous intraepithelial lesions (HSILs) (4). HSILs may eventually lead to invasive carcinoma and should be detected and treated early.

Screening programs based on Pap smear have contributed to a reduction in the incidence and mortality of cervical cancer (5). However, cytology shows variable sensitivities, suffers from subjectivity which depends on the skills of the observer, and cannot solve undetermined cytological changes (6). Moreover, $50 \%$ of invasive cervical cancers arise in women screened with existing cytological methodologies, suggesting the limits of effectiveness have been reached (7).

Several studies have established that infection with certain types of human papillomavirus (HPV) is the main cause for the development of cervical cancer $(8,9)$. HPVs are DNA viruses that infect mucosal and cutaneous epithelia and comprise more than 120 putative virus types. The high-risk (HR) types (HPV-16, -18, -31, -33, -35, -39, -45, -51, -52, -56, $58,-59,-68,-73$, and -82$)(10)$ can be identified in nearly all cervical carcinomas and their persistence is associated with 
progression to cervical disease $(9,11)$. Therefore, molecular methods for the detection and typing of HPV DNA have been proposed as an adjunct to the cytological screening programs (12). This strategy has provided more reassuring screening results due to the following findings: i) a very high negative predictive value (NPV) of the combined HPV DNA plus Pap smear, and ii) the higher sensitivity of HPV testing combined with the better specificity of Pap smear for detecting high-grade lesions allow that most of the small number of cases of HSILs missed by the HPV DNA test can be detected by Pap smear (12).

Many HPV DNA tests based on hybridization and amplification techniques have been developed, and only a few are commercially available. To date, the Hybrid Capture 2 Test (HC2) from Digene Corporation is the only test approved by the Food and Drug Administration (FDA) for HPV infection screening for women over age 30 in conjunction with the Pap smears. The HC2 assay is based on direct hybridization of nucleic acid targets and detects virtually all HR-HPV types, as well as most non-oncogenic (low-risk) HPV genotypes. Among detection tests using PCR, the MY09/11 HPV consensus primer system (13) is the most used for HPV DNA detection and typing (14-16) and has been considered a 'gold standard' for HPV testing. The MY09/11 primer set targets conserved sequences within the HPV L1 gene and can amplify and detect more than 25 of the HPV genotypes known to infect the genital mucosa (16). However, commercial assays for HPV DNA testing are expensive and practically inaccessible in developing countries where the majority of cases of cervical cancer occur. This is mainly due to the lack of regional biotechnological industries involved in the development of molecular assays for diagnostics. This limitation prevents the large-scale application of nucleic acid methods in most settings and prompt the development of reliable cost effective in-house assays.

Several aspects should be considered when developing an in-house assay for HPV DNA testing. Some of them are related to the general characteristics of the genital HPV infection. Although 40 HPV types are sexually transmitted, only 15 of them are associated to the development of cervical cancer (10). Among them, types 16, 18, 45, 31, 33, 52, 58, and 35 are the most frequent HPV types found in patients (10). Besides, most infections, including those which cause cytological abnormalities, are transient and resolve spontaneously. Uncommonly, an HPV infection will progress to a highgrade preinvasive lesion which typically contain HR-HPVs. At this stage, many of this high-grade lesions could progress to invasive cervical cancers (17). On the other hand, the extremely high analytical sensitivity of the PCR technique plays against test specificity because a high rate of positive results could be obtained in women who do not have significant histological abnormalities. Therefore, the main requirements of an in-house assay for HPV DNA testing should include a cutoff point that consider analytical and clinical parameters, a reproducible identification of genital HPV infection and high clinical sensitivity and specificity for the detection of lesions $\geq$ HSIL.

In this work, we present the L1HPVPCR, a PCR-based method for the detection and typing of five of the most prevalent HR-HPV types (HPV-16, -18, -31, -33, -35). The
L1HPVPCR assay combines amplification with the MY09/11 HPV consensus primer system, liquid hybridization of the PCR products with no radioactive probes and enzyme immunoassay analysis (EIA) for the detection of probe-amplicon hybrids. We also evaluated its performance and clinical utility as either an alternative method for primary screening or as adjunctive test to cytology in a group of women at risk of cervical cancer from Rosario city (Argentina).

\section{Materials and methods}

Cell lines and plasmids. The following cell lines were used as positive controls for optimization experiments: HeLa and CasKi cervical carcinoma cell lines, which contain 10-50 copies of HPV-18/cell (18) and 60-600 copies of HPV-16/cell (19), respectively. A cell line derived from monkey kidney cells, Vero cells, was used in all experiments as negative control. All cell lines were obtained from the Tissue Culture Service of the National Institute of Infectious Diseases 'Carlos G. Malbrán' (Buenos Aires, Argentina), grown as monolayer and passaged at regular intervals using standard cell culture techniques. After trypsinisation, cells were removed from the plastic, counted and aliquots of $4 \times 10^{6}$ cells $/ \mathrm{ml}$ were prepared. Each aliquot was resuspended in $1 \mathrm{ml}$ of lysis buffer $(1 \%$ Tween-20, 10 mM Tris-HCl, 1 mM EDTA pH 8.3 and $100 \mu \mathrm{g}$ of proteinase $\mathrm{K}$ ), and lysed overnight at $55^{\circ} \mathrm{C}$. After proteinase $\mathrm{K}$ inactivation at $95^{\circ} \mathrm{C}$ for $10 \mathrm{~min}$, cell lysates were stored at $-20^{\circ} \mathrm{C}$. In order to avoid variations in analytical sensitivity by DNA dilution, each dilution was prepared in Vero cell lysates at a concentration of 4,000 cells $/ \mu 1$. Five microliters of each dilution, corresponding to $200 \mathrm{ng}$ of DNA, were tested using the L1HPVPCR assay.

Recombinant clones for each HPV type were used as positive controls for HPV clinical samples screening. For HPV-16, we used a plasmid bearing the complete HPV-16 sequence; pHPV-35 was kindly supplied by Dr Aldo Venuti (Regina Elena Cancer Institute, Rome, Italy). MY11/09 PCR fragments from type HPV-18 derived from HeLa cells were purified using Wizard PCR Preps DNA Purification (Promega, Madison, USA) and cloned into pGEM-T-Easy vector (Promega). Positive controls for types HPV-31 and -33 were cloned from clinical samples generously provided by $\mathrm{Dr}$ Magdalena Grce (Ruder Boskovic Institute, Zagreb, Croatia) (20) using the procedure described above for HPV-18.

Human subjects and specimen collection. From April 2002 to June 2004, 127 women attending for cervical screening at the Division of Cervical Pathology of the Centenario Provincial Hospital were enrolled to participate in the study. The mean age of the women was 30.6 years (age range: 18-68 years). Eleven women in this group were HIV-infected. The Division of Cervical Pathology received women that are derived from the Department of Gynecology due to cervical lesions at the gynecologic visit, abnormalities in previous Pap smears, and/or HIV infection, and therefore are considered a risk population for cervical cancer development. Participants signed a written informed consent and interviews were performed to address risk factors for HPV infection. Institutional Review Board approval was obtained from each institution involved in the study. 
All women underwent a complete gynecologic examination that included cervical cytology (Pap smear), colposcopy, and HPV DNA testing. For cervical cytology, an Ayres spatula was used to collect exocervical cells, and a conical cytobrush (Cervicep, Buenos Aires, Argentina) to collect endocervical cells. Once Pap smear was performed, the cytobrush was kept in $0.8 \mathrm{ml}$ PBS solution for HPV DNA testing. Bioptic samples were taken in abnormal areas and were histologically analyzed in the Department of Pathology, School of Medicine, Rosario National University (Argentina). A positive screening test in cytology was defined as a Pap smear showing LSIL or higher. Besides, the criteria used for diagnosis in both histology and cytology was the worst morphology observed. For example, an LSIL morphology with a focus of HSIL was considered HSIL.

The referral was a combined diagnosis based on the results of cytology, colposcopy and morphology of biopsies. Women were considered positive in the combined diagnosis if they had HPV-induced cytological changes in Pap smears, lesions on colposcopy and atypical morphology in direct punch biopsies. In case of disagreement among these 3 screening tests, positivity was determined with a positive result in the biopsy specimen taken by colposcopy. Women were considered to be free of cervical disease if they were negative for Pap smear and colposcopy or if biopsies taken by colposcopy were negative.

Sample processing. For HPV DNA testing, 127 samples of cervical cells were collected from the cytobrush the same day of collection and washed several times with PBS to eliminate mucus. Cells were then pelleted by centrifugation and, depending on the pellet size, resuspended in 200-500 $\mu \mathrm{l}$ of lysis buffer and processed as described above.

In addition, HPV detection was performed in 16 paraffinembedded histological sections of the cervix derived from 16 women, corresponding to 4 HSIL diagnoses and 12 invasive squamous cervical carcinomas. Formalin-fixed, paraffinembedded tissues were deparaffinized using a standard method (21) and lysed as described above. DNA was then extracted and purified by conventional procedures, resuspended in 25-200 $\mu 1$ of TE buffer (10 mM Tris-HCl, $1 \mathrm{mM}$ EDTA pH 8.3) and stored at $-20^{\circ} \mathrm{C}$. Quality of cell lysates and extracted DNA was assessed on HPV-negative samples by PCR amplification of the B-globin gene as described (22).

HPV detection and typing. PCR technique was optimized for the amplification of a 450-bp fragment of the L1 gene from most HPV mucosal types, with primers MY11 and biotinylated MY09 (13,16). Forty-five microliters of a PCR mixture containing $50 \mathrm{mM} \mathrm{KCl}, 10 \mathrm{mM}$ Tris- $\mathrm{HCl} \mathrm{pH} 8.5,2.5 \mathrm{mM}$ $\mathrm{MgCl}_{2}, 0.2 \mathrm{mM}$ deoxynucleoside triphosphates, $0.4 \mu \mathrm{M}$ of each primer (TIB Molbiol, Genoa, Italy), and 2.5 U of Taq DNA polymerase (Invitrogen, Buenos Aires, Argentina) were dispensed into microtubes placed on ice. A 5- $\mu 1$ aliquot of cell lysate was added to the PCR mixture and amplified in a Mastercycler Personal (Eppendorf, Hamburg, Germany). After 2 min of initial denaturation at $95^{\circ} \mathrm{C}$, the following 35 cycles were performed: 5 cycles $\left(20 \mathrm{sec}\right.$ at $94^{\circ} \mathrm{C}, 20 \mathrm{sec}$ at $56^{\circ} \mathrm{C}, 20 \mathrm{sec}$ at $\left.72^{\circ} \mathrm{C}\right)$ followed by 30 cycles $\left(20 \mathrm{sec}\right.$ at $94^{\circ} \mathrm{C}$, $20 \mathrm{sec}$ at $52^{\circ} \mathrm{C}, 20 \mathrm{sec}$ at $72^{\circ} \mathrm{C}$ ) with a final extension of $5 \mathrm{~min}$ at $72^{\circ} \mathrm{C}$. Each PCR run included the following controls: positive plasmidic controls (PC) for each HPV-type analyzed in this study (HPV-16, -18, -31, -33, -35), an HPV-negative control (NC) (Vero cells lysate) and a reagent control (RC) $\left(\mathrm{H}_{2} \mathrm{O}\right.$ instead of sample).

To identify HPV infection, biotinylated PCR products were hybridized to a generic probe, GPX (13), and detected colorimetrically. Five microliters of each PCR product were added to $120 \mu 1$ of hybridization buffer [5X SSC (1X SSC is $0.15 \mathrm{M} \mathrm{NaCl}, 0.015 \mathrm{M}$ sodium citrate)] containing 3 pmol of GPX which was synthesized with the insertion of a fluorescein molecule. The mixture was heated at $95^{\circ} \mathrm{C}$ for 5 min to denature the DNA duplex and then held at $40^{\circ} \mathrm{C}$ for $10 \mathrm{~min}$ to allow hybridization. Forty-five microliters of the hybridized product were transferred to a streptavidin-coated 96-well microtiter plate (Nunc, Miami, USA) for hybrid capture. Following incubation for $1 \mathrm{~h}$ at $37^{\circ} \mathrm{C}$, unbound components were removed by extensive washing. Captured products were detected with $100 \mu \mathrm{l}$ of conjugated solution [0.003 U of horseradish peroxidase-conjugated anti-fluorescein antibodies (Roche, Buenos Aires, Argentina), $100 \mathrm{mM}$ Tris- $\mathrm{HCl} \mathrm{pH} 7.5,150 \mathrm{mM} \mathrm{NaCl}$, and 3\% fetal calf serum (Gibco, Buenos Aires, Argentina)]. The microplate was incubated for $30 \mathrm{~min}$ at room temperature on a microplate shaker and, after a final wash, $100 \mu 1$ of tetramethylbenzidine solution (TMB) (Wiener Lab, Rosario, Argentina) were added to each well. After a 30-min incubation to allow color development, the reaction was stopped by the addition of $1 \mathrm{M}$ $\mathrm{H}_{2} \mathrm{SO}_{4}$. End-point optical density (OD) was read in a Stat-Fax 2100 microplate reader (Awareness Technology Inc., Palm City, USA) at $450 \mathrm{~nm}$, with a reference filter at $650 \mathrm{~nm}$.

Samples resulting HPV-positive with GPX generic probe were colorimetrically detected with specific fluoresceinated probes for HR-HPV types with the same protocol described for GPX. Probes used for typing were the following: MYB95/MYB133 (HPV-16), WDB74/MYB130 (HPV-18), MYB128/AG111 (HPV-31), MYB16/MYB64 (HPV-33) and MYB115/MY117 (HPV-35). All probes were previously described (13), with the exception of AG111 (F-ccaaaagccca aggaagatc). AG111 probe has a perfect match with the HPV-31 sequence and was designed to replace MYB92 (13), which presented 3 mismatches respect the current available sequences. Two oligonucleotide probes per type were used to avoid misclassification in typing, in a total amount of $4 \mathrm{pmol} /$ hybridization ( 2 pmol of each type specific probe). The hybridization temperatures for liquid hybridization of HPVspecific probes were $40^{\circ} \mathrm{C}$ for MYB95/MYB133 (HPV-16) and WDB74/MYB130 (HPV-18), and $55^{\circ} \mathrm{C}$ for MYB128/AG111 (HPV-31), MYB16/MYB64 (HPV-33) and MYB115/MY117 (HPV-35).

Statistical analysis. The kappa statistic (23) was used to assess reproducibility at different cutoff points. The cutoff points for the generic and HR-probes were selected using an univariate logistic regression model (24) with the true status (HSIL or cancer vs. LSIL or negative) as response variable, and the ratio of the observed and reference values (OD value of negative control) as explanatory variables. Each model was used to construct receiver operating characteristic curves (ROC) and the area under the curve (AUC) was estimated. 
Confidence intervals for the AUC were derived using bootstrap techniques (25). The sensibility and specificity of L1HPVPCR and Pap methods were compared using the McNemar test.

For ROC analysis, all samples were classified according to the combined diagnosis. The 'disease' group included a total of 28 samples, in which 16 were classified as HSIL, and 12 as invasive squamous cervical carcinomas. It is worth noting that DNA from paraffin-embedded tissues were also included to increase the number of pathological specimens. The 'healthy' group was constituted by 115 cervical lysates: 93 samples with negative cytology and 22 LSIL diagnoses. These criteria were based on our observation that low-grade lesions were typically transient and benign.

\section{Results}

Rationale and design of the HPV detection and typing system. The L1HPVPCR system was designed with a similar strategy to the assay previously applied for the detection of HIV $(26,27)$. The L1HPVPCR assay includes the following steps: i) HPV DNA amplification with primers MY11 and biotinylated MY09, ii) liquid hybridization of the biotin-labeled amplified products to the generic fluorescein-containing probe GPX, iii) hybrid capture into streptavidin-coated microplate wells, iv) colorimetric detection with an antifluorescein antibody conjugated with horseradish peroxidase and TMB cromogen, v) color production measurement in a microplate reader, vi) typing of the HPV-positive samples with typespecific probes for HPV-16, $-18,-31-33$ and -35 with the same procedure described above (items ii-v), and vii) $\beta$-globin detection of GPX-negative samples to control DNA integrity, the presence of DNA in adequate amounts, or amplification inhibitors.

Optimization of L1HPVPCR assay was achieved in two stages. At the first stage, initial conditions for PCR and colorimetric detection were optimized using plasmid controls and cell lines. The criteria used for the optimization were to choose those conditions which resulted in the greatest OD values for the positive controls with minimum OD values for the negative ones after colorimetric detection. At a second stage, and to adjust conditions for clinical samples, further optimization was performed with specimens previously characterized as HPV positive or HPV negative (data not shown). The final conditions of the assay are described in the Materials and methods.

Evaluation of the LIHPVPCR assay performance. Considering that we lacked a molecular test to be used as a gold standard, the evaluation of the L1HPVPCR was carried out by fixing each parameter in the following order: determination of the analytical cutoff, followed by intra-laboratory reproducibility analysis, analytical sensitivity and specificity. Once these intrinsic parameters were established, clinical parameters and the performance of the assay were determined.

First, we explored cutoff points that ensure good analytical sensitivity of the assay as well as provide clinically relevant results. To set a cutoff point at analytical level, we analyzed the reproducibility of the L1HPVPCR using the generic probe at different thresholds. We chose randomly 41 clinical samples (27 HPV-positive and $14 \mathrm{HPV}$-negative) based on

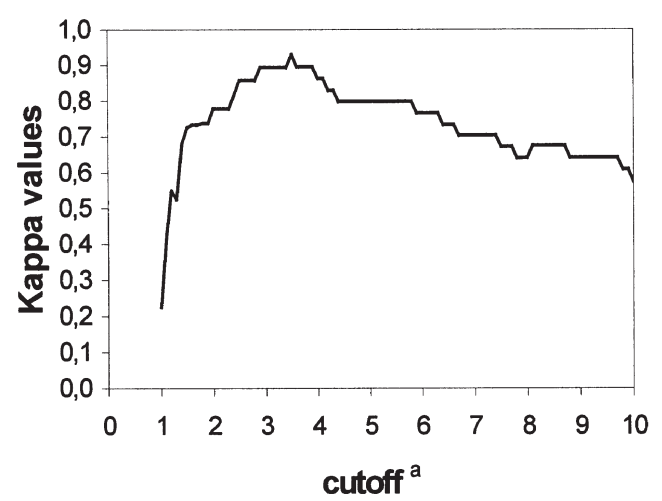

Figure 1. Analysis of the analytical cutoff by kappa values. ${ }^{a} n$ times the mean OD values of negative controls.

the results obtained in the first run of the L1HPVPCR with generic probe. These samples were tested 2 more times in independent experiments and kappa values were calculated for each threshold. Fig. 1 shows kappa values vs. cutoff values ranging from 1 to 10 times the mean OD value of HPV-negative controls. The best kappa value was obtained at a cutoff level of 3.5 times the mean OD value of HPV-negative controls $(\mathrm{K}=0.93)$. However, we chose a cutoff of 2.5 times $(\mathrm{K}=0.80)$ because screening with generic probe defines HPV positivity and we preferred to gain sensitivity at this assay step.

In view of the cutoff selected for the generic probe we analyzed the intra-laboratory reproducibility calculating the percent agreement considering all the 41 tested samples and the percent agreement considering only HPV-positive results at some runs (29 samples). As shown in Table I, the percent agreement at 3 runs for generic HPV positivity was $86.2 \%$ if only 29 specimens were considered, and $90.2 \%$ for all 41 specimens.

Analytical sensitivity of the L1HPVPCR assay was determined for types 16 and 18 using serial dilutions of plasmids bearing HPV-16 or HPV18 sequences, respectively, in a range of $10-10^{4}$ copies/reaction for each HPV type. HPV detection was performed with GPX generic probe to determine sample positivity or negativity. Sensitivity for HPV-16 and HPV-18 was 10 copies/reaction and 50 copies/reaction, respectively (data not shown). These results were confirmed using serial dilutions of CaSki and HeLa cells, considering the number of HPV genomes contained in each cell line, previously reported $(18,19)$.

To test analytical specificity, positive controls for HPV-16, $-18,-31,-33$ and -35 were analyzed with the L1HPVPCR assay in separate reactions and detected with generic and specific probes for each type. No cross-reaction was observed between types and none of the negative controls gave signals above the cutoff value with the HPV specific or generic probes (data not shown).

Lastly, a ROC analysis was performed for generic and HR-probes to evaluate cutoff values at a clinical level. ROC curves plot sensitivity against the positivity rate for women without disease (1-specificity) at different thresholds of test positivity, and allow to evaluate test performance from the AUC. As shown in Fig. 2, the ROC curve calculated with 
Table I. Intra-laboratory reproducibility of HPV amplification with generic detection.

\begin{tabular}{|c|c|c|c|c|c|c|}
\hline & \multicolumn{4}{|c|}{ Results in the 3 Runs ${ }^{a}$} & \multicolumn{2}{|c|}{ Agreement $(\%)^{\mathrm{b}}$} \\
\hline & $+/+/+$ & $+/+/-$ & $+/-/-$ & $-/-/-$ & 1 & 2 \\
\hline HPV DNA detection & 25 & 1 & 3 & 12 & $90.2(37 / 41)$ & $86.2(25 / 29)$ \\
\hline
\end{tabular}

${ }^{\mathrm{a}}+$, positive assay result; -, negative assay result. ${ }^{\mathrm{b}}$ Two measurements of percent agreement were calculated: 1 , including specimens that had negative results in all 3 runs; 2, excluding specimens that had negative results in all 3 runs.

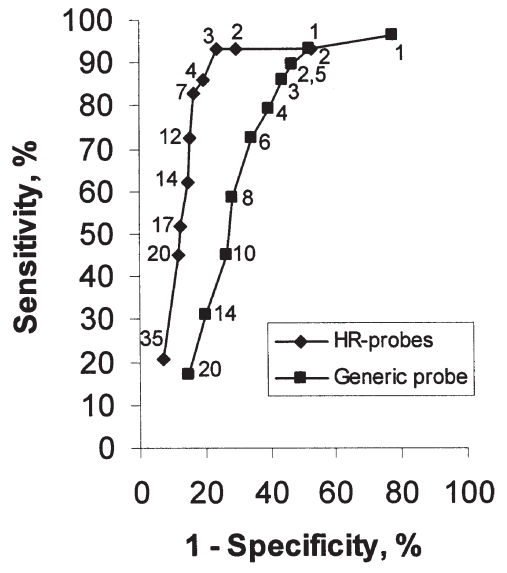

Figure 2. ROC analysis of the L1HPVPCR assay for the detection of HSIL or higher using generic or HR-probes. The numbers on the points means ' $n$ ' times the mean OD values of negative controls.

generic probe had an inflection point within $86-93 \%$ sensitivity and $48-56 \%$ specificity, corresponding to cutoff values between 2 and 3 times the mean OD value of HPV-negative controls. Therefore, the optimal balance between sensitivity (89.7\%) and specificity $(53.5 \%)$ was obtained when the L1HPVPCR assay cutoff value was $\sim 2.5$ times the mean OD value of HPV-negative controls. These findings confirmed the analytical cutoff point previously determined for the assay with the generic probe. As expected, the performance of the L1HPVPCR assay for the detection of HSILs was low, with an AUC of 0.704 (95\% CI: 0.39, 0.82).

When the ROC curve was calculated using the L1HPVPCR with HR-probes, the inflection point was within $83-93 \%$ sensitivity and $76-83 \%$ specificity, corresponding to cutoff values between 3 and 7 times the mean OD value of HPVnegative controls (Fig. 2). In this case, the optimal balance between sensitivity $(86.2 \%)$ and specificity (80.6\%) was obtained when the L1HPVPCR assay cutoff level was $\sim 4$ times the mean OD value of HPV-negative controls. Also, the performance of the L1HPVPCR assay for the detection of HSILs with HR-probes was very good, as shown by an AUC of 0.843 (95\% CI: 0.730, 0.934).

Although the data obtained with generic probe indicated a lower specificity $(53.5 \%)$ in respect to the HR-probes $(80.6 \%)$, this analysis constitutes the first screening step of the assay that determine sample positivity or negativity for HPV sequences.
HPV-DNA testing with the L1HPVPCR assay. The analysis of analytical sensitivity, reproducibility and ROC curves allowed to establish reliable cutoff points for the application of the L1HPVPCR assay as a tool for HPV DNA testing. Therefore, we used the L1HPVPCR assay to test a population at risk of cervical cancer and to evaluate its utility as either an alternative method for primary screening or as adjunctive test to cytology. With that purpose, HPV DNA testing was performed on the residual cervical cells that remained after the cytology slides were prepared.

A total of 127 cervical lysates from 127 women recruited at the Division of Cervical Pathology of Centenario Provincial Hospital were tested. Table II shows HPV typing results and the distribution of HPV sequences and genotypes according to the severity of histopathological cervical lesions determined by the referral (combined diagnosis). HPV sequences were detected in $51 \%$ of women, being HPV-16 the most prevalent type found, followed by HPV-18 and -33 (6\% prevalent both) and HPV-31. All women were negative for type HPV-35 and $24 \%$ of them were HPV-positive but negative for the HR-types detected in this work. Multiple infections were found in 3 women: 2 had negative diagnoses and one belonged to the LSIL category. Among negative women, a dual (HPV-16 + HPV-33) and a triple infection (HPV-18 + HPV-31 + HPV-33) were found. An infection with 3 types (HPV-18 + HPV-31 + HPV-33) was also present in a woman of the LSIL category. The prevalence of HPV infection was $43 \%$ in the healthy group and it increased with the increasing severity of the histopathological lesions, being 68 and $83 \%$ in the LSIL and HSIL categories, respectively. The same trend was observed for types 16 and 33, which in negative women showed 10 and $3 \%$ prevalences, respectively, increasing further in LSIL (18 and 9\%, respectively) and HSIL (58 and 17\%, respectively) categories.

We further compared the HPV prevalence in samples derived from HIV-negative and HIV-positive women. In HIVpositive, HPV DNA was found in $82 \%(9 / 11)$ of the women and typed as high-risk in $73 \%(8 / 11)$ of them, while in HIVnegative women, HPV DNA was present in $48 \%$ (56/116) and typed as HR in $23 \%(27 / 116)$ of them. Although the number of HIV-infected women included was small, these results were statistical significant using Fisher's exact test ( $p=0.03$ for HPV DNA and $\mathrm{p}=0.001$ for HR-types) and confirmed the higher prevalence of HR-HPV types in HIV-positive women.

To further explore the clinical implication of HPV DNA testing in respect to Pap smear we analyzed the rate of 
Table II. Distribution of HPV genotypes according to histopathological cervical lesions.

\begin{tabular}{lccccccc}
\hline & HPV & \multicolumn{5}{c}{ No. (\%) of HPV type-specific positivity } \\
\cline { 3 - 8 } Subjects (no.) & $\begin{array}{c}\text { posivity } \\
\text { No. (\%) }\end{array}$ & 16 & 18 & 31 & 33 & 35 & Untyped $^{\text {a }}$ \\
\hline All women (127) & $65(51)$ & $20(16)$ & $7(6)$ & $6(5)$ & $7(6)$ & 0 & $30(24)$ \\
Negative (93) & $40(43)$ & $9(10)$ & $6(6)$ & $3(3)$ & $3(3)$ & 0 & $22(24)$ \\
LSIL (22) & $15(68)$ & $4(18)$ & $1(4)$ & $2(9)$ & $2(9)$ & 0 & $8(36)$ \\
HSIL (12) & $10(83)$ & $7(58)$ & 0 & $1(8)$ & $2(17)$ & 0 & 0 \\
\hline
\end{tabular}

${ }^{\text {a } V i r a l ~ g e n o t y p e s ~ o t h e r ~ t h a n ~} 16,18,31,33,35 .{ }^{\text {b}}$ Type-specific numbers and percentages include multiple type infections.

Table III. Combined diagnosis vs. DNA HPV testing or Pap smears.

\begin{tabular}{|c|c|c|c|c|}
\hline & \multicolumn{4}{|c|}{ No. (\%) of samples with the following results } \\
\hline & \multicolumn{4}{|c|}{ Combined diagnosis ${ }^{\mathrm{a}}$} \\
\hline & Negative & LSIL & HSIL & Total \\
\hline \multicolumn{5}{|c|}{$\begin{array}{l}\text { L1HPVPCR } \\
\text { (generic probe) }\end{array}$} \\
\hline Positive & $40(43)$ & $15(68)$ & $10(83)$ & $65(51)$ \\
\hline Negative & $53(57)$ & 7 (32) & $2(17)$ & $62 \quad(49)$ \\
\hline \multicolumn{5}{|c|}{$\begin{array}{l}\text { L1HPVPCR } \\
\text { (HR-probes) }\end{array}$} \\
\hline Positive & $18(19)$ & $7(32)$ & $10(83)$ & $35 \quad(28)$ \\
\hline Negative & $75(81)$ & $15(68)$ & $2(17)$ & $92 \quad(72)$ \\
\hline \multicolumn{5}{|l|}{ Pap test } \\
\hline Positive & $6(6)$ & $13(59)$ & $8(67)$ & $27(21)$ \\
\hline Negative & 87 (94) & $9(41)$ & $4(33)$ & 100 (79) \\
\hline Total & $93(73)$ & $22(17)$ & $12(10)$ & $127(100)$ \\
\hline
\end{tabular}

${ }^{a}$ Negative, negative for intraepithelial lesions; LSIL, low-grade intraepithelial lesions; HSIL, high-grade intraepithelial lesions.

detection with each test in relation to the combined diagnosis. As shown in Table III, only 57\% of the women included in the negative group had concordant results of HPV DNA testing and the referral. In contrast, $19 \%$ of women in this group had HR-HPV sequences and $24 \%$ of them had untyped HPV sequences. In the LSIL group, $68 \%$ of women were HPV-positive, $32 \%$ with HR-HPV types, while $83 \%$ of women included in the HSIL group were HPVpositive with HR-HPV types. The rate of detection of Pap smear for cervical abnormalities was lower than HPV DNA testing for HSIL lesions (67 vs. $83 \%$, respectively) but cytology showed a higher concordance with the combined diagnosis (94\%) in respect to HPV DNA testing in the negative group (57\% with generic probe and $81 \%$ with HR-probes).
Table IV. Performance characteristics of L1HPVPCR assay, Pap smears and the combination of both screening methods in detecting HSIL or higher.

\begin{tabular}{lcccc}
\hline $\begin{array}{l}\text { Screening } \\
\text { tests }\end{array}$ & $\begin{array}{c}\text { Sensitivity } \\
(\%)\end{array}$ & $\begin{array}{c}\text { Specificity } \\
(\%)\end{array}$ & $\begin{array}{c}\text { NPV } \\
(\%)\end{array}$ & $\begin{array}{c}\text { PPV } \\
(\%)\end{array}$ \\
\hline $\begin{array}{l}\text { L1HPVPCR } \\
\text { (HR-probes) }\end{array}$ & 83.3 & 78.3 & 97.8 & 28.6 \\
Pap test & 66.7 & 83.5 & 96.0 & 29.6 \\
$\begin{array}{l}\text { L1HPVPCR } \\
\text { (HR-probes) }\end{array}$ & 100 & 66.1 & 100 & 23.5 \\
+ Pap test & & & & \\
\hline
\end{tabular}

Finally, to evaluate the clinical utility of HPV DNA testing as either an alternative method for primary screening or as adjunctive test to cytology, we analyzed the performance characteristics of the L1HPVPCR assay, Pap smear and the combination of both screening methods for the detection of cytological abnormalities for HSIL or higher (Table IV). HPV DNA testing showed higher sensitivity than conventional cytology (83.3 vs. $66.7 \%$, respectively) but cytology showed better specificity than HPV testing (83.5 vs. $78.3 \%$, respectively). When results of both screening methods were considered together, a sensitivity and NPV of $100 \%$ were obtained although with lower specificity than cytology alone $(83.5$ vs. $66.1 \%)$. Both screening tests showed positive predictive values (PPV) bellow $30 \%$ and this parameter did not increase when results from both tests were considered together. However, and due to the small number of subjects included in our study, differences in sensitivity, specificity, NPV and PPV were not statistically significant.

\section{Discussion}

The recognition that infection with HPV is essential for the development of cervical cancer has been enormously important $(11,28)$. This knowledge and accumulated evidence suggest that incorporation of HPV testing could be suitable for primary 
screening in conjunction with cytology, management and triage of women with low-grade disease, and test of cure of treatment of high-grade disease (7). Indeed, HPV testing has potential advantages in the developing world where the infrastructure, training opportunities and expertise to maintain high quality cytology screening are lacking. However, the introduction of HPV DNA testing in these countries has been delayed due to the inaccessibility of high performance and cost effective diagnostic nucleic acid methods. We strongly believe that the major challenge for the future of developing countries is the creation of own resources to solve regional problems. Accordingly, we developed a user-friendly system to allow accurate HPV DNA detection and typing with inexpensive instrumentation that could be performed without sophisticated reagents in almost any laboratory. The newly developed L1HPVPCR method is based on consensus PCR amplification of a conserved sequence of $30 \mathrm{HPV}$ genital genotypes and on the typing of 5 HR-HPVs by liquid hybridization to nonradioactive probes followed by an EIA immunoassay.

L1 consensus primer PCR systems, particularly the MY09/ 11 primer systems, along with a broad-spectrum of detection methods such as gel electrophoresis or dot blotting techniques have been widely used to study the natural history of HPVs and their role in the development of genital cancer $(5,14)$. The L1HPVPCR method described here includes a universal protocol for the detection of PCR products in streptavidincoated 96-well microtiter plates that have been applied for the screening of other viruses $(26,27)$ and can be used for the detection of any other biotin-labeled amplicons. Besides, the EIA protocol has several advantages over the conventional dot blot method, such as microplates are easily handled than the dot blot membrane, washing is carried out in shorter times, lower background levels are obtained, and a quantifiable cutoff can be set to determine sample positivity or negativity. The last advantage is essential to reduce the subjectivity of the analysis and to eliminate some of the associated potential experimental errors. In particular, the cutoff point of an assay to be used for HPV testing should allow to differentiate between HPV-positive and HPV-negative samples as well as to provide clinically relevant results. We set a cutoff point for generic probe of 2.5 times the mean OD values of HPV-negative controls $(\mathrm{K}=0.80)$, based on the assay reproducibility (Fig. 1). In general, a kappa value above 0.75 indicates excellent agreement, between 0.40 and 0.75 indicates fair to good agreement, and $<0.40$ represents poor agreement beyond chance (23). This results were further confirmed by ROC analysis. As shown in Fig. 2, the optimal balance between sensitivity $(89.7 \%)$ and specificity $(53.5 \%)$ was obtained when the L1HPVPCR assay cutoff value using generic probe was $\sim 2.5$ times the mean OD value of HPV-negative controls. The low performance for the generic detection of HPV sequences (AUC $=0.704 ; 95 \%$ CI: $0.39,0.82$ ) was due to the fact that both low-risk and HR-HPVs types are detected with the generic probe, but only the last ones are associated with cancer development.

In a similar way, we set a cutoff value of 4 times the mean OD values of HPV-negative controls for HR-probes (Fig. 2). At this cutoff point, the performance of the L1HPVPCR assay was very good, as shown by the AUC $(0.843$; 95\% CI: 0.730 , $0.934)$. Therefore, the clinical meaning of the results obtained with the L1HPVPCR is based on testing with HR-probes which improve specificity $(80.6 \%)$, maintaining high sensitivity $(86.2 \%)$.

We used cell lines, plasmids, and clinical samples to explore the analytical performance of the newly developed L1HPVPCR method. The detection limit of L1HPVPCR was 10 and 50 genome copies of HPV-16 and HPV-18, respectively, demonstrating similar analytical sensitivity to others HPV detection techniques, including PCR-EIA (10-200 viral copies) (29-31). Furthermore, the method was specific and precise, with an overall intra-laboratory reproducibility of $90.2 \%$ (Table I). Respect to HC2 test, unique molecular method approved by the FDA for HPV testing, the L1HPVPCR assay was 10-100 times more sensitive and showed similar reproducibility (32).

Further, we used the L1HPVPCR to estimate the prevalence of HPV infection in a group of women at risk of cervical cancer from Rosario, the second large urban center in Argentina (Table II). HPV sequences were present in 51\% and at least one of the five HR-HPV types analyzed in this study were found in $28 \%$ of them. The rate of HPV detection and the prevalence of types 16 and 33 increased in parallel with the increasing severity of cytological abnormalities. HPV-18 behaved in the opposite way, being more frequently found in negative women (6\%) than LSIL (4\%), while none in the HSIL group presented this type. The fact that HPV-18 genome fully integrates into the host genome in pre-invasive lesions confers major aggressiveness in terms of rapid progression (33) and could explain its lower detection rate in the endocervical cells used for DNA HPV testing. Indeed, we found mixed infections with types 16 and 18 in several tissue samples from women that presented only HPV-16 in cervical lysates collected at the same time of the biopsy (data not shown). Based on this observation, it would be of interest to test deeper (biopsies) vs. superficial (cervical lysates) tissues from the same subject to corroborate the real prevalence of HPV-18 in cervical lesions.

Among HPV-infected women, HPV-16 was the most prevalent type, followed by HPV-18 and -33, and HPV-31. A higher prevalence of HR-HPV types in HIV-positive women (73\%) in respect to the HIV-negative ones (23\%) was also found. These results give us an approximation of the prevalence of HR-HPV types in a population at risk for developing cervical cancer in Rosario City (province of Santa Fe), from where no epidemiological data has been previously reported. Although we limited our analysis to five HR-HPV genotypes, the approach will be extended to other HR-genotypes (HPV-45, 52 and -56) for which specific oligoprobes and positive controls are now available at our laboratory.

HPV-35 was absent in our group, in contrast with other studies reporting this type more prevalent in Argentina (34) and Latin America than in the other regions (10). Moreover, other studies performed in different cities of Argentina such as La Plata (province of Buenos Aires) (35), Concordia (province of Entre Ríos) (34), Posadas (province of Misiones) (36) and Corrientes (province of Corrientes) (37) showed high intra-country variation in the prevalence of HPV infection and in the distribution of HPV genotypes. These findings reinforce the need to determine the regional variation in the distribution of HR-HPV types, specially to predict the effect of vaccines on the incidence of infection. 
We explored the clinical utility of HPV DNA testing respect to Pap smear using a combined diagnosis of cytology, histology and colposcopy as gold standard (Table III). Cytology showed a higher concordance with the referral in the negative group but $19 \%$ of HR-HPV DNA sequences were found among them. These HPV test-positive/Pap smear-negative women have became a major challenge for HPV testing, because transient anogenital HPV infections are very common in young and sexually active women $(17,38)$. However, persistent infections with HR-HPV types predispose women to a significantly increased risk for the development of SIL and cervical cancer $(17,39)$ and should be monitored more frequently. On the other hand, women resulting negative for both HR-HPV DNA and Pap smear have better prognostic assurance against risk of future HSIL than 3 subsequent negative conventional Pap tests and may safely allow 3-year screening intervals for such low-risk women (12). However, this issue remains to be assessed in our population.

The rate of detection of HPV DNA testing for HSIL lesions was higher than Pap smear (Table III). It is worth to consider that women analyzed in this study belong to low socioeconomic strata and their access to health centers is limited. Therefore, good quality and high sensitive tests are needed to detect those at greatest risk of high-grade cervical lesions. In our study, the combination of HPV DNA testing and Pap smear showed sensitivity and NPV of $100 \%$, although with a lower specificity than Pap smear alone (Table IV). This decrease in specificity could potentially be offset by greater protection from neoplastic progression. However, these results should be assessed with a larger number of subjects.

In conclusion, available evidence indicates great potential for HPV DNA testing within the cervical screening programs based on cytology. It is important to note that HPV testing only could be assessed with standardized and high performance HPV tests. The L1HPVPCR assay described and evaluated in this study provides a tool for accurate HPV DNA testing and could be applied in regions where no commercial tests are available.

\section{Acknowledgements}

The authors thank Dr Emilia Rivadeneira from the Centers of Disease Control and Prevention (USA) for helpful discussions and manuscript revision. This research was funded by a Fogarty International Center/NIH grant through the AIDS International Training and Research Program at Mount Sinai School of Medicine-Argentina Program (Grant \#5D43 TW0010137) and the Agencia Nacional de Promoción Científica y Tecnológica (Grant \#05-09894).

\section{References}

1. World Health Organization: Cervical cancer screening in developing countries: report of a WHO consultation. World Health Organization, Geneva (ISBN 9241545720), 2002.

2. Ferlay J, Bray F, Pisani P and Parkin DM: Globocan 2000: cancer incidence, mortality and prevalence worldwide, version 1.0. IARC CancerBase no. 5. IARC Press, Lyons, 2001.

3. Lewis MJ: A situational analysis of cervical cancer in Latin America and the Caribbean. Washington, DC, PAHO:2004, 2004.
4. Solomon D, Davey D, Kurman R, et al: The 2001 Bethesda System: terminology for reporting results of cervical cytology. JAMA 287: 2114-2119, 2002.

5. Coutlée F, Mayrand MH, Provencher D and Franco E: The future of HPV testing in clinical laboratories and applied virology research. Clin Diagn Virol 8: 123-141, 1997.

6. Cuzick J, Meijer CJ and Walboomers JM: Screening for cervical cancer. Lancet 351: 1439-1440, 1998.

7. Cuzick J: Role of HPV testing in clinical practice. Virus Res 89: 263-269, 2002.

8. Zur Hausen H: Papillomavirus infections - a major cause of human cancer. Biochim Biophys Acta 1288: F55-F78, 1996.

9. Zur Hausen H: Papillomavirus in human cancers. Proc Assoc Am Physicians 6: 581-587, 1999.

10. Muñoz N, Bosch FX, Sanjosé S, et al: Epidemiologic classification of human papillomavirus types associated with cervical cancer. N Engl J Med 348: 518-527, 2003.

11. Walboomers JMM, Jacobs MV, Manos MM, et al: Human papillomavirus is a necessary cause of invasive cervical cancer worldwide. J Pathol 189: 12-19, 1999.

12. Lorincz AT and Richart RM: Human papillomavirus ADN testing as an adjunct to cytology in cervical screening programs. Arch Pathol Lab Med 127: 959-968, 2003.

13. Manos MM, Ting Y, Wright DK, Lewis AJ, Broker T and Wolinski SM: Use of polymerase chain reaction amplification for the detection of genital human papillomaviruses. Cancer Cells 7: 209-214, 1989.

14. Bosch FX, Manos MM, Muñoz N, et al: Prevalence of human papillomavirus in cervical cancer: a worldwide perspective. J Natl Cancer Inst 87: 796-802, 1995.

15. Nobbenhuis MA, Walboomers JMM, Helmerhorst TJ, et al: Relation of human papillomavirus status to cervical lesions and consequences for cervical cancer screening: a prospective study. Lancet 354: 20-25, 1999.

16. Ting Y and Manos MM: Detection and typing of genital human papillomaviruses. In: PCR Protocols: A Guide to Methods and Applications. Innis MA, Gelfand DH, Sninsky JJ and White TJ (eds). Academic Press, San Diego, pp356-367, 1990.

17. Syrjänen K and Syrjänen S: Papillomavirus Infections in Human Pathology. John Wiley \& Sons, Inc., New York, NY, 2000.

18. Jones HW Jr, McKusick VA, Harpe PS and Wuu K-D: The HeLa cell and a reappraisal of its origin. Obstet Gynecol 38: 945-959, 1971.

19. Pattillo RA, Hussa RO, Story MT, Ruckert ACF, Shalaby MR and Mattingly RF: Tumor antigen and human chorionic gonadotropin in CaSki cells: a new epidermoid cervical cancer cell line. Science 196: 1456-1458, 1977.

20. Grce M, Husnjak K, Bozikov J, et al: Evaluation of genital human papillomavirus infections by polymerase chain reaction among Croatian women. Anticancer Res 21B: 579-584, 2001.

21. Wright DK and Manos MM: Sample preparation from paraffinembedded tissues. In: PCR Protocols: A Guide to Methods and Applications. Innis MA, Gelfand DH, Sninsky JJ and White TJ (eds). Academic Press, San Diego, pp153-156, 1990.

22. Saiki RK, Bugawan TL, Horn GR, Mullis KB and Erlich HA: Analysis of enzymatically amplified $\beta$-globin and HLA DNA with allele-specific oligonucleotide probes. Nature 324: 163-166, 1986.

23. Fleiss JL: Statistical Methods for Rates and Proportions. 2nd edition. John Wiley, New York, pp211-232, 1981.

24. Agresti A: Categorical Data Analysis. 2nd edition. John Wiley \& Sons, Inc., Hoboken, New Jersey, pp165-195, 2002.

25. Efron B and Tibshirani R: An Introduction to the Bootstrap. Chapman \& Hall, New York, 1993.

26. McDermott JL, Giri AA, Martini I, et al: HIV DNA in peripheral blood mononuclear cells correlates with antiretroviral therapy efficacy. J Clin Microbiol 37: 2361-2365, 1999.

27. Gariglio R, Taborda MA, Bortolozzi R, et al: Non-conventional virological markers in HIV-infected patients: T-HIV DNA, 2LTR-HIV DNA and HIV RNA. Medicina 64: 419-428, 2004.

28. Bosch FX, Lorincz A, Muñoz N, Meijer CJ and Shah KV: The causal relation between human papillomavirus and cervical cancer. J Clin Pathol 55: 244-265, 2002.

29. Jacobs MV, Snijders PJF, van Den Brule AJC, Helmerhorst TJM, Meijer CJLM and Walboomers JMM: A general primer GP51/GP61-mediated PCR-enzyme immunoassay method for rapid detection of 14 high-risk and 6 low-risk human papillomavirus genotypes in cervical scrapings J Clin Microbiol 35: 791-795, 1997. 
30. Hart KW, Williams OM, Thelwell N, Fiander AN, Brown T, Borysiewicz LK and Gelder CM: Novel method for detection, typing, and quantification of human papillomaviruses in clinical samples. J Clin Microbiol 39: 3204-3212, 2001.

31. Swan DC, Tucker RA, Tortolero-Luna G, et al: Human papillomavirus (HPV) DNA copy number is dependent on grade of cervical disease and HPV type. J Clin Microbiol 37: 1030-1034, 1999.

32. Schiffman MH, Kiviat NB, Burk RD, et al: Accuracy and interlaboratory reliability of human papillomavirus DNA testing by hybrid capture. J Clin Microbiol 33: 545-550, 1995.

33. Cullen AP, Reid R, Campion M and Lorincz A: Analysis of the physical state of different human papillomavirus DNAs in intraepithelial and invasive cervical neoplasm. J Virol 65: 606-612, 1991.

34. Matos E, Loria D, Amestoy GM, et al: Prevalence of human papillomavirus infection among women in Concordia, Argentina: a population-based study. Sex Transm Dis 30: 593-599, 2003.

35. Abba MC, Gomez MA and Golijow CD: Human papillomavirus genotype distribution in cervical infections among woman in $\mathrm{La}$ Plata, Argentina. Rev Argent Microbiol 35: 74-79, 2003.
36. Tonon SA, Picconi MA, Zinovich JB, et al: Human papillomavirus cervical infection and associated risk factors in a region of Argentina with a high incidence of cervical carcinoma. Infect Dis Obstet Gynecol 7: 237-243, 1999.

37. Deluca GD, Lucero RH, De Civetta M, Vicente L, De Gorodner OLZ, Schelover E and Alonso JM: Human papillomavirus genotypes in women with cervical cytological abnormalities from an area with high incidence of cervical cancer. Rev Inst Med Trop S Paulo 46: 9-12, 2004

38. Ho GYF, Bierman R, Beardsley L, Chang CJ and Burk RD: Natural history of cervicovaginal papillomavirus infection in young women. N Engl J Med 338: 423-429, 1998.

39. Koutsky LA, Holmes KK, Critchlow CW, et al: A cohort study of the risk of cervical intraepithelial neoplasia grade 2 or 3 in relation to papillomavirus infection. N Engl J Med 327: 1272-1278, 1992. 